\title{
Active Phase Correction of Compact, High Resolution Silicon Photonic Arrayed Waveguide Gratings
}

\author{
M. Gehl, D. Trotter, A. Starbuck, A. Pomerene, A. L. Lentine, C. DeRose \\ Applied Photonic Microsystems, Sandia National Laboratories, Albuquerque, NM, 87123
}

\begin{abstract}
We demonstrate compact silicon photonic arrayed waveguide gratings with channel spacing down to $1 \mathrm{GHz}$ using active phase correction. The relative phase of each path within the device is directly measured using an interferometer, and two methods of phase optimization are implemented and compared.
\end{abstract}

Arrayed waveguide gratings(AWG) provide the integrated photonic equivalent of a ruled grating, and offer useful functionality for a range of applications from optical communications to RF signal processing [1,2]. Unlike a freespace grating, the AWG disperses light among a large number of waveguides with incrementally increasing lengths. The light from each waveguide is then recombined at a slab waveguide, where it coherently interferes. In the ideal device the light is spatially dispersed by wavelength and an array of output waveguides collects different slices, or channels, of the optical spectrum.

RF photonic signal processing can offer a great advantage in terms of size, weight, power and cost compared to traditional electronic methods. In order to accomplish RF signal processing with AWGs, one needs channels spaced narrowly in frequency. This necessitates rather long path length differences between each AWG waveguide. In low index silica based systems this results in devices with very large footprints [3]. Higher index materials, such as silicon, not only provide shorter physical paths, but also permit tighter waveguide bends, allowing waveguides to be folded into a smaller area. The major drawback to high index contrast materials is the difficulty in controlling the optical phase of light passing through each waveguide. Small perturbations in the size or index of the of waveguide result in much larger fluctuations in the phase of guided light as the index contrast increases [4]. As a result, the output of the AWG becomes random unless some additional measure is taken to correct optical phase errors. Methods for correcting the optical phases include both passive techniques such as UV irradiation [5] and active devices such as thermal phase shifters.

In this work, we present measurements of silicon AWGs with active thermal phase shifters. The devices have channel spacing from $50 \mathrm{GHz}$ down to $1 \mathrm{GHz}$. We directly measure the optical phases of the multiple paths within the device and demonstrate two methods of active phase correction. For thermal phase shifters, thermal crosstalk and temperature stability become key issues which we also address.

We fabricate AWG devices using a CMOS compatible process on SOI wafers with a 3 micron buried oxide and a $230 \mathrm{~nm}$ silicon device layer. The devices have 11 input waveguides which are split into 35 dispersing waveguides, before recombining into 11 output waveguides. Several device variations with channel spacing of 50,10 and $1 \mathrm{GHz}$ were designed, fabricated and measured. By tightly winding waveguides, these devices are kept to a very compact footprint. The largest device, with $1 \mathrm{GHz}$ channel spacing, fits within a $1.2 \mathrm{~cm}^{2}$ chip.

Each of the 35 AWG dispersing waveguides includes a thermal phase shifter consisting of a $\mathrm{N}+$ doped silicon resistor in close proximity to the waveguide. A series of NI DAQ PXI-6704 boards are used to precisely drive between 0 and $20 \mathrm{~mA}$ through each phase shifter. The temperature, and as a result of the thermo-optic effect, the optical phase of each waveguide, is expected to vary linearly with the applied power.

Temperature stability is extremely important for these devices. Due to the thermo-optic effect, a change in temperature generates a phase shift in each of the 35 waveguides proportional to the waveguide length. This creates a linear phase shift across the waveguides, which results in a shift in the transmitted wavelength, measured to be on the order of $11 \mathrm{GHz} /{ }^{\circ} \mathrm{C}$. To control this, we implement a TEC sample mount. For the most sensitive measurements the device is placed in a sealed enclosure which allows stabilization to better than $0.005^{\circ} \mathrm{C}$.

Optical transmission spectra are collected using an Agilent tunable laser source and single channel photodetector. For the measurement of optical phase, a swept wavelength Mach Zehnder Interferometer(MZI) setup is used [6]. A reference MZI provides a clock signal which triggers sample acquisition, while the device is inserted into one arm of the second MZI. The interferogram generated by the second MZI is collected and digitally processed in order to retrieve the relative phase of each path within the device.

While the MZI provides direct access to phase information, it can be challenging to implement in practical applications. For this reason, we developed an optimization method based on the measurement of the transmitted intensity of a fixed wavelength laser from one input waveguide to one output waveguide. The transmitted intensity can be intuitively thought of as the addition of 35 phasors, one from each of the dispersing waveguides. In the ideal device, each of the phasors points along the same direction, constructively adding; however, the random phases of 
the un-optimized device cause a random walk of these phasors. By varying a single phase shifter from 0 to $2 \pi$, the associated phasor will rotate about a circle, causing the intensity to oscillate with the same period. One can then choose the phase setting which maximizes the intensity and repeat the process for each of the remaining phase shifters. This process essentially straightens the random walk of the phasors. The oscillation in intensity is related to the amplitude of the phasor, which is on the order of $1 / 35$ times the input intensity. Therefore, this method requires precise intensity measurements in order to accurately set the phases. Using this method, we have demonstrated better than $18 \mathrm{~dB}$ of contrast between the peak transmission and the next highest side-lobe of a device with $10 \mathrm{GHz}$ channel spacing, as shown in fig. 1a. In fig. $1 \mathrm{~b}$ we compare this to the optimization of the device using direct phase measurement with the MZI, showing slightly better side-lobe suppression.
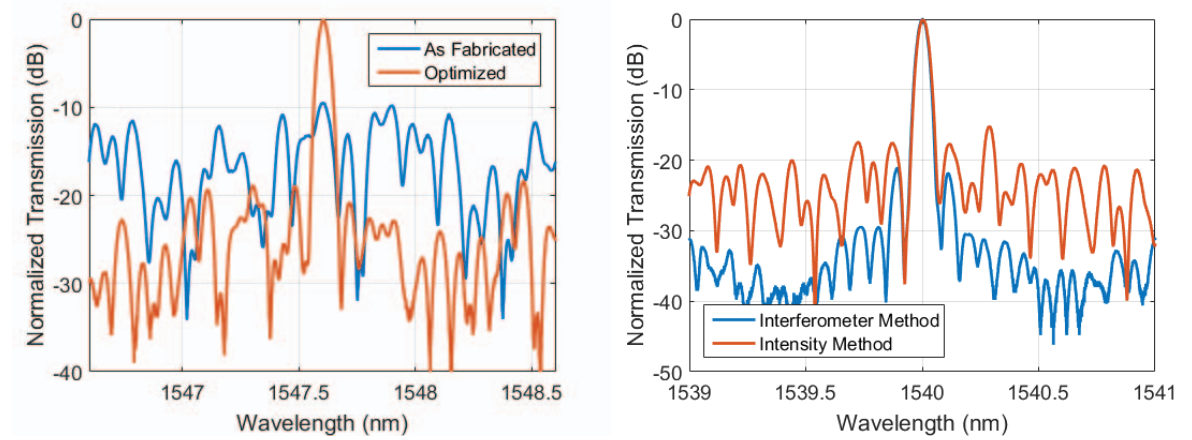

Figure 1 - (left) Comparison of the AWG transmission as fabricated with random phase errors and with active phase correction optimized using only the intensity of a transmitted fixed wavelength, and (right) comparison of the intensity optimization method and interferometer method.

Due to the temperature sensitivity of the device and the thermal crosstalk between phase shifters, the intensity based optimization technique becomes more difficult to implement for $1 \mathrm{GHz}$ channel spacing. For this reason, we use the MZI method to optimize this device as shown in fig. 2. In fig. 2a the residual phase of each path is shown, and in fig. $2 \mathrm{~b}$ the transmission through a single output waveguide is shown along with the location of the other channel outputs represented by dashed lines. Thermal issues prevented perfect optimization; however, we were still able to achieve greater than $18 \mathrm{~dB}$ contrast between the peak and side-lobes.
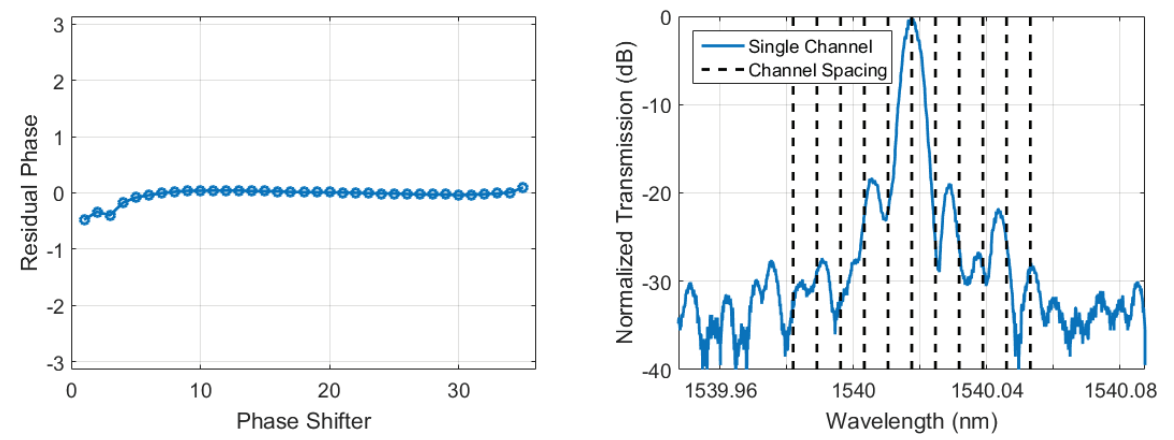

Figure 2 - (left) Residual phase error in the 35 waveguides of an AWG with $1 \mathrm{GHz}$ channel spacing after active phase correction, and (right) measured optical spectrum of the same device.

In conclusion we have demonstrated a compact silicon photonic arrayed waveguide grating with channel spacing as low as $1 \mathrm{GHz}$ and footprint less than $1.2 \mathrm{~cm}^{2}$. We achieve optimized transmission through active phase correction provided by integrated thermal phase shifters. We compare two methods of phase optimization and show greater than $18 \mathrm{~dB}$ of contrast between the peak transmission and the next highest side-lobe.

Sandia is a multiprogram laboratory operated by Sandia Corporation, a Lockheed Martin Company, for the United States Department of Energy's National Nuclear Security Administration under contract DE-AC04-94AL85000.

[1] S. Takashina, et. al., "Wavelength-Tunable Filters Utilizing Arrayed Waveguide Gratings for Colorless/Directionless/Contentionless Optical Signal Drop in ROADMs." IEEE Photonics Journal 7, 1-11 (2015).

[2] N.K. Fontaine, et. al., "Compact $10 \mathrm{GHz}$ loopback arrayed-waveguide grating for high-fidelity optical arbitrary waveform generation," Opt. Lett. 33, 1714-1716 (2008).

[3] R. Rudnick, et. al., "One GHz Resolution Arrayed Waveguide Grating Filter with LCoS Phase Compensation," Opt. Fiber Comm., paper Th3F.7. (2014).

[4] T. Goh, et. al., "Estimation of waveguide phase error in silica-based waveguides." J. Lightwave Tech. 15, 2107-2113 (1997).

[5] K. Takada, et. al. "1-GHz-spaced 16-channel arrayed-waveguide grating for a wavelength reference standard in DWDM network systems." $J$. Lightwave Tech. 20, 850-853 (2002).

[6] E. D. Moore, "Advances in swept-wavelength interferometry for precision measurements." PhD diss., University of Colorado (2011). 